\title{
Desalinhamento cambial, volatilidade cambial e crescimento econômico: uma análise para a economia brasileira (1995-2011)
}

\author{
Exchange rate misalignment, exchange rate volatility and \\ economic growth: an analysis for the Brazilian economy
}

FLÁVIO VILELA VIEIRA

ADERBAL OLIVEIRA DAMASCENO *

\begin{abstract}
RESUMO: O objetivo deste trabalho é investigar a importância do desalinhamento cambial e da volatilidade cambial para o crescimento da economia brasileira no período de 1995 a 2011. As evidências sugerem: i) o desalinhamento cambial é relevante para explicar o crescimento econômico da economia brasileira e subvalorização cambial (sobrevalorização cambial) estimula (desestimula) o crescimento econômico; ii) a volatilidade cambial é relevante para explicar o crescimento econômico da economia brasileira e maior (menor) volatilidade cambial desestimula (estimula) o crescimento econômico; iii) mudança na taxa de investimento e a taxa de crescimento das exportações têm coeficientes estimados positivos no modelo de crescimento. Como recomendação de política, sugere-se a manutenção de uma taxa real de câmbio em um nível competitivo e com baixa volatilidade. PALAVRAS-CHAVE: Desalinhamento Cambial; Volatilidade Cambial; Crescimento Econômico. JEL Classification: O40; F31; C26.
\end{abstract}

ABSTRACT: The goal of this work is to investigate the relevance of exchange rate misalignment and volatility for the Brazilian growth rate for the period of 1995 to 2011. The evidence suggest: $i)$ the exchange rate misalignment is relevant to explain economic growth for the Brazilian economy and exchange rate under depreciation (over appreciation) foster (mitigates) economic growth; ii) exchange rate volatility is relevant to explain economic growth for the Brazilian economy and higher (lower) exchange rate volatility mitigates (foster) economic growth; iii) changes in the investment rate and the growth rate of exports have positive estimated coefficients for the growth model. As a recommendation policy, the suggestion is to keep the real exchange rate at a competitive level with low volatility. KEYWORDS: Exchange Rate Misalignment; Exchange Rate Volatility; Economic Growth. JEL Classification: O40; F31; C26.

\footnotetext{
* Instituto de Economia - Universidade Federal de Uberlândia, Pesquisador CNPq e FAPEMIG, flaviovieira@ufu.br; Instituto de Economia - Universidade Federal de Uberlândia, Pesquisador CNPq e FAPEMIG, damasceno@ie.ufu.br. Os autores agradecem o apoio financeiro recebido do CNPq e da FAPEMIG. Submetido: 27/Maio/2014: Aprovado: 4/Setembro/2015.
} 


\section{INTRODUÇÃO}

Observa-se crescente interesse na literatura sobre o papel do desalinhamento cambial para o crescimento econômico. Três abordagens se destacam: a associada a Williamson (1990), Krueger (1983) e Edwards (1998), segundo a qual o desalinhamento cambial é prejudicial ao crescimento; a associada a Eichengreen (2008) e Rodrik (2008), os quais argumentam que subvalorização cambial estimula o crescimento e sobrevalorização cambial desestimula o crescimento; por fim, a macroeconomia estruturalista do desenvolvimento, associada a Bresser-Pereira (2009a, 2009b, 2012) e Bresser-Pereira e Gala (2010), considera uma taxa de câmbio competitiva fundamental para o crescimento.

Outra vertente da literatura, a qual tem recebido menos atenção, foca não no papel do desalinhamento cambial, mas na relevância da volatilidade cambial para o crescimento econômico, considerando, dentre os canais de transmissão, comércio, investimento, desemprego e produtividade. Emerge da análise da literatura empírica sobre o papel da taxa de câmbio para o crescimento econômico dois conjuntos de resultados: subvalorização cambial estimula o crescimento econômico, e este efeito é mais acentuado para as economias em desenvolvimento; há evidências de que maior volatilidade cambial desestimula o crescimento econômico.

O objetivo deste trabalho é investigar o papel do desalinhamento cambial e da volatilidade cambial para o crescimento da economia brasileira no período de 1995 a 2011. Para tanto, são adotados os seguintes procedimento metodológicos: construção de uma série de taxa de câmbio real efetiva e estimação da taxa de câmbio real efetiva de equilíbrio; cálculo do desalinhamento cambial, da subvalorização cambial, da sobrevalorização cambial e de duas medidas de volatilidade cambial; estimações de equações de crescimento, incluindo entre as variáveis explanatórias a medida de desalinhamento cambial, de subvalorização cambial, de sobrevalorização cambial e as medidas de volatilidade cambial.

A taxa de câmbio real efetiva de equilíbrio e o desalinhamento cambial são estimados por meio da abordagem comportamental da taxa real de câmbio (BEER) de Clark e MacDonald (1999). O desalinhamento cambial é definido como desvios da taxa de câmbio real efetiva corrente em relação à taxa de câmbio real efetiva de equilíbrio e há sobrevalorização (subvalorização) cambial quando o valor da taxa de câmbio real efetiva corrente for menor (maior) do que o valor da taxa de câmbio real efetiva de equilíbrio, implicando valor do desalinhamento cambial negativo (positivo). Ademais, as duas medidas de volatilidade cambial para a série de taxa de câmbio real efetiva corrente foram calculadas por meio de um modelo GARCH e desvio-padrão móvel.

Esse trabalho é oportuno no contexto atual da economia brasileira, considerando o debate sobre a tendência de apreciação cambial, o fenômeno da desindustrialização e os determinantes da desaceleração recente da taxa de crescimento, para o qual as referências importantes são Oreiro e Feijó (2010), Bresser-Pereira (2008) e Palma (2005). Esse trabalho contribui para o debate ao apresentar evidências sistemáticas de que subvalorização cambial (sobrevalorização cambial) esti- 
mula (desestimula) o crescimento e maior (menor) volatilidade cambial desestimula (estimula) o crescimento. Como implicação normativa, emerge desses resultados a recomendação de manutenção da taxa de câmbio real em nível competitivo e com baixa volatilidade.

O trabalho encontra-se dividido em três seções além desta introdução e das considerações finais. A segunda seção é dedicada à revisão teórica e empírica sobre desalinhamento cambial, volatilidade cambial e crescimento econômico. A terceira seção refere-se aos procedimentos metodológicos para o cálculo do desalinhamento, da volatilidade cambial e para a especificação do modelo de crescimento. Por fim, a quarta seção sistematiza e discute os resultados econométricos.

\section{DESALINHAMENTO CAMBIAL, VOLATILIDADE CAMBIAL E CRESCIMENTO ECONÔMICO}

\section{Desalinhamento Cambial e Crescimento Econômico}

A taxa de câmbio real não desempenha um papel central na teoria do crescimento econômico convencional. Tanto o modelo neoclássico de crescimento quanto os modelos de crescimento endógeno representam economias fechadas. O debate contemporâneo sobre a relação entre taxa de câmbio e crescimento econômico permite distinguir ao menos duas visões no âmbito da abordagem convencional (Mbaye, 2013):

A primeira, denominada "Consenso de Washington", sustenta que o valor da taxa de câmbio deve ser fixado em um nível consistente com o equilíbrio interno e externo. ${ }^{1}$ Os desvios da taxa de câmbio em relação a este nível de equilíbrio, desalinhamento cambial, estão associados a algum tipo de desequilíbrio macroeconômico, independentemente da direção do desalinhamento, sendo, portanto, prejudicial ao crescimento econômico (Williamson, 1990; Krueger, 1983; Edwards, 1998).

A segunda considera que subvalorização cambial favorece o crescimento econômico por meio de dois canais: estimula a acumulação de capital ao encorajar altos níveis de poupança e investimento; estimula a produtividade ao aumentar a participação do setor de bens comercializáveis, considerando a existência de externalidades positivas associadas a este setor. Ademais, argumenta-se que sobrevalorização cambial desestimula o crescimento econômico e subvalorização cambial estimula o crescimento econômico (Eichengreen, 2008; Rodrik, 2008). ${ }^{2}$

\footnotetext{
${ }^{1}$ Nessa abordagem, equilíbrio interno é considerado o nível de produto compatível com pleno emprego e baixa inflação, e a poupança líquida gerada a esse nível de produto deve ser igual ao saldo sustentável em conta-corrente, que não é necessariamente zero nessa abordagem e define o equilíbrio externo.

${ }^{2}$ Para o cálculo da taxa de câmbio de equilíbrio e desalinhamento cambial, Rodrik (2008) e Eichengreen (2008) utilizam a abordagem da Paridade do Poder de Compra (PPC), estendida para levar em
} 
De acordo com Bresser-Pereira (2009a, 2009b, 2012) e Bresser-Pereira e Gala (2010), a macroeconomia estruturalista do desenvolvimento considera uma taxa de câmbio competitiva fundamental para o crescimento econômico: dado o progresso técnico em curso, o crescimento econômico é função da taxa de investimento; a taxa de investimento, por sua vez, depende da existência de oportunidades de lucros; uma taxa de câmbio competitiva estimula os investimentos orientados para a exportação. ${ }^{3}$

Os estudos analisados na sequência sistematizam algumas das contribuições para a literatura empírica sobre desalinhamento cambial e crescimento econômico. Um dos trabalhos pioneiros sobre a relação entre desalinhamento cambial e crescimento econômico é Edwards (1989), argumentando que o desalinhamento cambial tende a criar distorções nos preços relativos dos bens comercializáveis e não comercializáveis, resultando em alocações não ótimas de recursos entre os setores da economia, com impactos adversos sobre o crescimento econômico. Razin e Collins (1997) investigam a relação entre desalinhamento cambial e crescimento econômico para 93 países desenvolvidos e em desenvolvimento durante o 1975-1992 e encontram evidências de que sobrevalorização cambial muito elevada parece estar associada a crescimento econômico mais lento; subvalorização cambial moderada a elevada (mas não muito elevada) parece estar associada a crescimento econômico mais rápido.

Aguirre e Calderón (2005) investigam a relação entre desalinhamento cambial e crescimento econômico para um conjunto de 60 países durante o período 1965-2003. As evidências reportadas sugerem que o efeito do desalinhamento cambial sobre o crescimento econômico não é linear, o que significa que, quando a subvalorização da taxa real de câmbio for demasiado elevada o impacto sobre o crescimento econômico é negativo, mas quando a subvalorização da taxa real de câmbio é pequena ou moderada, pode estimular o crescimento econômico. Gala e Lucinda (2006) desenvolvem uma análise para uma amostra de 58 países durante o período 1960-1999. As evidências reportadas corroboram o argumento de que a taxa real de câmbio subvalorizada (sobrevalorizada) está associada a maiores (menores) taxas de crescimento econômico.

Rodrik (2008) investiga a relação entre desalinhamento cambial e crescimento econômico para 184 países durante o período 1950-2004. O principal resultado

consideração o efeito Balassa-Samuelson. Essa abordagem consiste em estimar uma equação para a taxa de câmbio real controlando para diferenças de renda per capita entre países. O desalinhamento cambial é a diferença entre a taxa de câmbio real corrente e a taxa de câmbio real dada pela equação estimada.

${ }^{3}$ Conforme Bresser-Pereira (2012, p. 10): "Uma taxa de câmbio competitiva é fundamental para o desenvolvimento econômico porque ela funciona como uma espécie de interruptor de luz que 'liga' ou 'desliga' as empresas tecnológica e administrativamente competentes à demanda mundial. Uma taxa de câmbio competitiva estimula os investimentos orientados para a exportação e aumenta correspondentemente a poupança interna. Uma taxa de câmbio competitiva é aquela que se situa no 'equilíbrio industrial', ou seja, que corresponde à taxa de câmbio necessária para que empresas que utilizem tecnologia no estado da arte mundial sejam internacionalmente competitivas". Marconi (2012) apresenta uma metodologia para o cálculo da taxa de câmbio de equilíbrio industrial e estimativas para o Brasil. 
empírico é que o crescimento econômico é maior em países com taxas reais de câmbio mais subvalorizadas e o efeito é linear e similar para ambos, subvalorização e sobrevalorização, o que implica que uma taxa real de câmbio subvalorizada estimula o crescimento econômico e uma taxa real de câmbio sobrevalorizada desestimula o crescimento econômico. A magnitude e significância estatística do coeficiente estimado para a subvalorização da taxa real de câmbio são maiores nos países em desenvolvimento, e a explicação apresentada pelo autor é a existência de fragilidade institucional e falhas de mercado mais severas nesse grupo de países.

Berg e Miao (2010) desenvolvem uma análise empírica sobre desalinhamento cambial e crescimento econômico, com propósito de comparar a visão de Rodrik (2008) e a visão do "Consenso de Washington". O principal resultado é que ambas as visões sobre o papel do desalinhamento cambial para o crescimento econômico são observacionalmente equivalentes para as principais regressões de crescimento. Sugerem a existência de um problema de identificação, dado que os determinantes do desalinhamento cambial são também prováveis determinantes do crescimento econômico. Contudo, confirmam que subvalorização estimula o crescimento econômico e sobrevalorização desestimula o crescimento econômico.

Vieira e MacDonald (2012) investigam a relação entre desalinhamento cambial e crescimento econômico para um conjunto de 90 países no período 1980-2004. As evidências indicam que subvalorização cambial estimula o crescimento econômico, e esse efeito é mais acentuado para os países em desenvolvimento. Mbaye (2013) desenvolve uma análise sobre a relação entre desalinhamento cambial e crescimento econômico para uma amostra de 72 países desenvolvidos e em desenvolvimento no período 1970-2008. As evidências sugerem que subvalorização cambial estimula o crescimento econômico e sobrevalorização cambial desestimula o crescimento econômico. Ademais, a importância do canal produtividade é maior de que a importância do canal acumulação de capital.

Eichengreen (2008) explicita os possíveis canais por meio dos quais a taxa real de câmbio pode influenciar o crescimento econômico e argumenta favoravelmente à manutenção da taxa de câmbio real efetiva em nível competitivo, contanto que não esteja associada a maior volatilidade da taxa de câmbio real efetiva. Especificamente, Eichengreen (2008) explicita os seguintes canais de transmissão subjacentes à relação entre desalinhamento cambial e crescimento econômico:

i) Uma taxa de câmbio real efetiva subvalorizada incentiva uma mudança na composição da produção por meio da alocação de recursos em direção ao setor de bens manufaturados comercializáveis e estimula o nível de renda, considerando o maior nível de produtividade no setor de bens manufaturados comercializáveis em relação à agricultura;

ii) Esse processo pode continuar durante um período considerável sem se deparar com retornos decrescentes, como experimentado na agricultura. Ademais, pode continuar sem resultar em queda de preços na medida em que a demanda externa é elástica, ao contrário da situação com bens não comerciáveis, onde a demanda é puramente interna e relativamente inelástica;

iii) Ele, assim, permite que a estrutura de produção seja desconectada da es- 
trutura de consumo. Se maior nível de renda e mais rápido crescimento econômico derem suporte a um maior nível de poupança, então será possível financiar um maior nível de investimento por meio da utilização de recursos domésticos;

iv) Por fim, se learning-by-doing ou transferência tecnológica é relativamente rápida no setor de bens manufaturados comercializáveis, então existirão estímulos adicionais para o crescimento econômico.

\section{Volatilidade Cambial e Crescimento Econômico}

Outra vertente da literatura sobre taxa de câmbio e crescimento econômico, foca na relevância da volatilidade cambial. A relação entre volatilidade cambial e crescimento econômico tem sido abordada pela literatura por meio de diferentes perspectivas e canais, tais como comércio, investimento, desemprego e produtividade (Eichengreen, 2008). Ademais, como destaca Eichengreen (2008), a literatura sobre volatilidade da taxa de câmbio real efetiva e crescimento econômico destaca que um aumento da volatilidade desestimula o comércio e o investimento, que são relevantes para o crescimento econômico. Outra parte da literatura centrada no argumento da ocorrência de problemas relacionados a descasamento de balanço e fragilidade financeira destaca que mudanças bruscas (maior volatilidade) na taxa de câmbio podem estar associadas a consequências financeiras adversas, em especial quando da ocorrência de crises cambiais em que ocorre significativa elevação da volatilidade cambial e mudanças nas reservas internacionais, sendo que ambos acabam tendo importantes custos que comprometem o crescimento econômico.

Cushman (1986) e Peree e Steinherr (1989) mostram teoricamente que maior volatilidade cambial tem efeitos adversos sobre o comércio, enquanto Viaene e De Vries (1992) encontram frágil relação entre as duas variáveis. Por outro lado, Franke (1991) e Sercu e Vanhulle (1992) constroem modelos teóricos que mostram que o comércio pode ser beneficiado pela volatilidade cambial. Caballero e Corbo (1989) e Peree e Steinherr (1989) encontram evidências de que a volatilidade cambial afeta adversamente o comércio, enquanto efeitos positivos são encontrados por Franke (1991), Sercu e Vanhulle (1992), Doyle (2001) e Bredin et al. (2003).

Quanto à relação entre volatilidade cambial e investimento, Campa e Goldberg (1995) concluem que a volatilidade cambial influencia adversamente o investimento utilizando dados para os Estados Unidos, mas não encontra tal evidência quando dados para o Canadá são utilizados. Darby et al. (1999) reporta evidências de que a volatilidade cambial tem efeito adverso sobre o investimento. Resultados semelhantes foram reportados por Bleaney e Greenaway (2001) e Serven (2002).

Considerando a relação entre volatilidade cambial e desemprego, Belke e Kaas (2004) sugerem que a volatilidade cambial reduz o crescimento do emprego. Aghion et al. (2009), utilizando dados para 83 países durante o período 1960-2000, encontram evidências de que a volatilidade cambial desestimula o crescimento da produtividade, mas apenas em países com mercados financeiros subdesenvolvidos.

Dollar (1992) investiga a relação entre volatilidade cambial e crescimento 
econômico para uma amostra de 95 países em desenvolvimento durante o período 1976-1985 e encontra evidências de que a volatilidade cambial desestimula o crescimento econômico. Bosworth et al. (1996) analisa uma amostra de 88 países ao longo do período 1960-1992 e encontram evidências de que a volatilidade cambial desestimula o crescimento econômico, via efeito adverso sobre o crescimento da produtividade total dos fatores.

Bleaney and Greenaway (2001) analisam uma amostra de 14 países da África Subsaariana no período 1980-1995 e não encontram relação significativa entre volatilidade cambial e crescimento econômico. Resultado similar foi encontrado por Ghura e Grennes (1993) para 33 países da África Subsaariana. Ghosh et al. (1997) não encontram nenhuma relação significativa entre volatilidade cambial e crescimento econômico para uma amostra de 140 países durante o período 1960-1990. Vieira et al. (2013) analisam a relação entre volatilidade cambial e crescimento econômico para uma amostra de 82 países desenvolvidos e em desenvolvimento no período 1970-2009. As evidências reportadas indicam que uma maior volatilidade cambial desestimula o crescimento econômico.

\section{PROCEDIMENTOS METODOLÓGICOS}

\section{Cálculo do Desalinhamento Cambial}

A abordagem comportamental da taxa real de câmbio (BEER) de Clark e MacDonald (1999) não se baseia em nenhum modelo específico de taxa de câmbio, não impõe nenhuma estrutura normativa e permite obter medida de taxa de câmbio real de equilíbrio e desalinhamento cambial por meio de tratamento estatístico rigoroso. ${ }^{4}$ Clark e MacDonald (1999) especificam uma equação em forma reduzida para o comportamento da taxa de câmbio real em função dos fundamentos relativos:

$$
\text { reer }_{t}=f\left(\text { tot }_{t}, \text { bs }_{t}, \text { divida }_{t}, r_{t}-r_{r}^{*}, n f a_{t}\right)
$$

Essa é a taxa de câmbio real de equilíbrio comportamental, onde tot $t_{t}$ é termos de troca, $b s_{t}$ é a razão entre o preço de bens não comercializáveis e comercializáveis e capta o efeito Balassa-Samuelson, divida $a_{t}$ é a dívida pública, $r t-r_{r}^{*}$ é o diferencial

\footnotetext{
${ }^{4}$ MacDonald (2000), MacDonald e Dias (2007) e Égert et al. (2006) argumentam que a elaboração de uma abordagem adequada para a estimação da taxa de câmbio real de equilíbrio e mensuração de desalinhamento cambial necessita reconhecer explicitamente a existência de determinantes reais da taxa de câmbio real, os fundamentos. Duas abordagens reconhecem os determinantes reais da taxa de câmbio real: i) a abordagem comportamental; ii) a abordagem do equilíbrio interno e externo. No âmbito dessa última abordagem, três modelos se destacam: a taxa de câmbio de equilíbrio fundamental (FEER) de Williamson (1983, 1994); a taxa de câmbio real natural (NATREX) de Stein (1994, 1995, 2002); o equilíbrio macroeconômico (IMF) de Isard e Faruqee (1998) e Faruqee, Isard e Masson (1999). Uma característica da abordagem do equilíbrio interno e externo é a substancial estrutura normativa imposta na definição de equilíbrio interno e externo, conforme apresentada na nota de rodapé 1 .
} 
de juros e $n f a_{t}$ é ativo externo líquido. O efeito esperado de cada variável sobre a taxa de câmbio real é indicado pelo sinal de cada derivada parcial:

$$
\begin{aligned}
& \text { dreer }_{t} / \text { Jtot }_{t}<0 ; \text { dreer }_{t} / \partial b s_{t}<0 ; \text { Jreer }_{t} / \text { ddivida }_{t}>0 ; \text { Jreer }_{t} / \partial\left(r_{t}-r_{r}^{*}\right)<0 ; \\
& \text { dreer } r_{t} / \partial n f a_{t}<0
\end{aligned}
$$

Conforme Égert et al. (2006), a estimação da taxa de câmbio real de equilíbrio comportamental procede em três etapas:

i) Estimar a relação estatística de longo prazo entre a taxa de câmbio real e os fundamentos, por meio de análise de cointegração;

ii) Identificar os valores de longo prazo dos fundamentos. Isso pode ser feito usando as abordagens de Hodrick e Prescott (1997) e Beveridge e Nelson (1981);

iii) Os valores de longo prazo dos fundamentos são substituídos na relação estimada. O desalinhamento total é a diferença entre o valor ajustado e atual da taxa de câmbio real.

Neste trabalho, a relação de longo prazo para a taxa de câmbio real é estimada por meio do Vetor de Correção de Erro (VEC) de Johansen (1995). Os valores de longo prazo dos fundamentos são calculados por meio do filtro de Hodrick e Prescott (1997), doravante denominado filtro HP. Os dados utilizados neste trabalho abrangem o período 1994T3 a 2011T4. Todos os índices têm como ano-base $2005=100$. Para a construção do índice de taxa de câmbio real efetiva é necessário construir os pesos, os quais são utilizados para calcular os fundamentos relativos àqueles dos parceiros comerciais.

Para a construção dos pesos foram considerados os 20 principais parceiros comerciais do Brasil: Alemanha, Arábia Saudita, Argentina, Bélgica, Canadá, Chile, China, Coreia do Sul, Espanha, Estados Unidos, França, Holanda, Índia, Itália, Japão, México, Reino Unido, Rússia, Suíça, Venezuela. O peso do parceiro comercial $i$ no período $t$, foi calculado como a razão entre o comércio total do parceiro comercial $i$ no período $t$ com o Brasil e o comércio total dos vinte parceiros comerciais com o Brasil no período $t$ (Fonte: Construção a partir de dados do Direction of Trade Statistics, 2013).

As definições das variáveis utilizadas para calcular a taxa de câmbio de equilíbrio e o desalinhamento cambial são:

Taxa de Câmbio Real Efetiva (REER): É a média ponderada das taxas de câmbio reais bilaterais do Brasil em relação aos vinte parceiros comerciais (Fonte: International Financial Statistics, 2013; Banco Central de Chile, 2013; China Statistical Yearbook, 2013; European Central Bank, 2013). ${ }^{5}$

\footnotetext{
${ }^{5}$ A expressão para o cálculo da taxa de câmbio real efetiva é dada por: ${ }^{R E E R}=\prod_{i=1}^{20}\left(\frac{s_{p q i}^{i}}{p_{p q i}^{i}}\right)^{w}$ onde $S^{i}$ é a taxa de câmbio nominal do real em relação à moeda do parceiro comercial $i$, definida como unidade de moeda estrangeria por unidades de moeda doméstica, $p_{c p i}^{i}$ é índice de preço ao consumidor do parceiro comercial $i, p_{c p i}^{b}$ é o índice de preço ao consumidor do Brasil, e $w^{i}$ são os pesos. Da forma como definida,
} 
Termos de Troca (TOT): É a razão entre o índice de preço de exportação e índice de preço de importação no Brasil, relativa à média ponderada da razão entre índice de preço de exportação e índice de preço de importação dos vinte parceiros comerciais (Fonte: World Bank, 2013; International Financial Statistics, 2013).

Balassa-Samuelson (BS): É a razão entre o índice de preço ao consumidor e índice de preço ao atacado ou produtor do Brasil, relativa à média ponderada da razão entre o índice de preço ao consumidor e índice de preço ao atacado ou produtor dos vinte parceiros comerciais (Fonte: International Financial Statistics, 2013; Banco Central de Chile, 2013; China Statistical Yearbook, 2013; OECD, 2013).

Ativo Externo Líquido (NFA): É a diferença entre o estoque total de ativos e estoque total de passivos, como porcentagem do PIB (Fonte: Lane, Milesi-Ferretti, 2007; International Financial Statistics, 2013; World Economic Outlook, 2013).

Dívida Pública (DÍVIDA): É a dívida bruta do setor público do Brasil como porcentagem do PIB, relativa à média ponderada da dívida bruta do setor público dos vinte parceiros comerciais (Fonte: Historical Public Debt Database, 2012; Fiscal Monitor, 2012).

Diferencial de Juros (DIFJUROS): É a diferença entre a taxa real de juros do Brasil e a média ponderada da taxa real de juros dos parceiros comerciais (Fonte: International Financial Statistics, 2013).

A estimação do VEC envolveu inicialmente a elaboração dos testes de estacionariedade (ADF, PP, KPSS e DF-GLS) das séries de tempo para LREER, LTOT, LBS, LDIVIDA, NFA e DIFJUROS. Os resultados indicaram que todas as séries não são estacionárias e integradas de ordem 1, isto é, I(1), com exceção da série DIFJUROS que apenas no teste DF-GLS se mostrou não estacionária. Assim sendo, manteve-se a estimação do VEC para as seis variáveis em questão, sendo que o número de defasagens escolhido foi de duas. Os testes de cointegração pela estatística do Traço e Máximo Autovalor indicaram, respectivamente, a existência de dois e um vetor cointegrante. ${ }^{6}$ Ao final estimou-se um $\operatorname{VEC}(2)$ com um vetor cointegrante na especificação com intercepto, mas sem tendência na equação cointegrante e sem intercepto no VAR.

O Gráfico 1 apresenta o comportamento da taxa de câmbio real efetiva corrente e da taxa de câmbio real efetiva de equilíbrio (fundamentos com filtro HP) e o gráfico 2 apresenta o comportamento do desalinhamento cambial total. As evidências apresentadas sugerem: para o período 1996T1 a 1998T4 (câmbio rígido) o desalinhamento total médio foi de $-17,2 \%$, indicando sobrevalorização cambial; para o período pós-1999T1 (câmbio flexível) o desalinhamento total médio é de 7,4 \%, indicando subvalorização cambial; para o período pós-2008T3

um aumento no valor da taxa de câmbio nominal ou da taxa de câmbio real efetiva significa uma depreciação (desvalorização).

${ }^{6}$ Conforme Enders (2004, p. 354): "The results of the $\lambda_{\text {trace }}$ and $\lambda_{\max }$ tests can conflict. The $\lambda_{\max }$ test has the sharper alternative hypothesis. It is usually preferred for trying to pin down the number of cointegrating vectors". 
(crise financeira) o desalinhamento total médio é 9,3\%, indicando subvalorização cambial; e para 2011 o desalinhamento total médio é $-7,1 \%$, indicando sobrevalorização cambial. Taxa de Câmbio Corrente
e de Equilíbrio

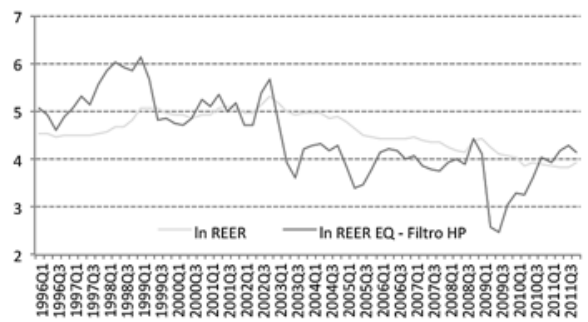

Desalinhamento Total (\%)

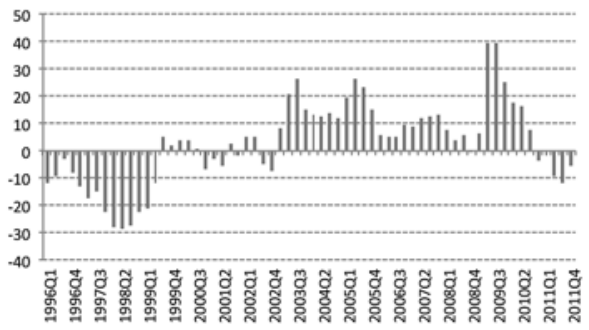

\section{Cálculo da Volatilidade Cambial}

A estimativa das duas medidas de volatilidade (VOLATCOND e DESVPADMOV) da taxa de câmbio real efetiva foi feita utilizando-se a série DLREER (primeira diferença do log da taxa de câmbio real efetiva). No caso de VOLATCOND estimou-se inicialmente um modelo autorregressivo com 4 defasagens, verificando a significância estatística para a primeira e a segunda defasagem, sugerindo a adoção de um modelo $\operatorname{AR}(2)$. Ao final estimou-se um modelo $\operatorname{AR}(2) \operatorname{GARCH}(1,1)$ para a obtenção da variância condicional e a partir desta o desvio-padrão condicional que é a proxy final utilizada. A segunda medida de volatilidade comumente utilizada na literatura empírica é o desvio-padrão móvel da série DLREER, que no cálculo envolveu 4 períodos (trimestres), resultando na medida DESVPADMOV.

\section{Especificação do Modelo de Crescimento e Métodos de Estimação}

Uma das razões para utilizar o Método dos Momentos Generalizado (GMM) é que enquanto as estimativas por Mínimos Quadrados Ordinários (MQO) frequentemente possuem problemas de autocorrelação serial, heterocedasticidade e não linearidade, o que é considerado comum nas séries de tempo macroeconômicas, o método GMM fornece estimadores consistentes para a análise de regressão. Cragg (1983) e Hansen (1982), ao analisar a técnica de estimação por GMM destacam que a análise das restrições sobreidentificadoras tem papel importante na seleção das variáveis instrumentais para melhorar a eficiência dos estimadores, e neste sentido um teste J é utilizado com o objetivo de testar as propriedades para a validade das restrições sobreidentificadoras.

Wooldridge (2001) enfatiza que a obtenção de estimadores mais eficientes do que MQO, ou Mínimos Quadrados em Dois Estágios (MQ2E), deve levar em consideração as restrições sobreidentificadoras, sendo que a matriz de ponderação na equação deve ser escolhida de tal forma a permitir que as estimações GMM sejam robustas à possível presença de heterocedasticidade e de autocorrelação. $\mathrm{O}$ 
autor destaca que os coeficientes estimados por GMM são consistentes apenas se as variáveis instrumentais utilizadas na análise forem exógenas. O autor enfatiza ainda que as estimativas GMM podem sofrer com problemas de amostras finitas, especialmente quando se adicionam muitas condições de momento que não adicionam muita informação à estimação.

Hansen (1982) ao analisar o estimador GMM para séries de tempo enfatiza que este permite a adição de condições de momento ao assumir que valores passados das variáveis explicativas, ou mesmo valores passados da variável dependente, são não correlacionados com o termo de erro, mesmo não estando presente diretamente no modelo.

As estimações dos modelos de crescimento por GMM foram feitas incluindo 5 defasagens das variáveis explicativas contidas em cada modelo, além da variação da taxa de câmbio real efetiva corrente e defasada também em 5 trimestres. $^{7}$

A estimação do modelo de crescimento para a economia brasileira com dados trimestrais de $1995 \mathrm{~T} 1$ a $2011 \mathrm{~T} 4$ tem por base as seguintes equações para investigar o papel do desalinhamento cambial, da subvalorização cambial, sobrevalorização cambial e da volatilidade cambial para o crescimento da economia brasileira:

$$
\begin{aligned}
& \text { TXCRESC }_{t}=\beta_{0}+\beta_{1} \text { TXCRESC }_{t-1}+\beta_{2} \text { DESALTOTAL }_{t}+\beta_{3} \text { TXINVEST }_{t} \\
& +\beta_{4} \text { VOLAT }_{t}+\beta_{5} \text { TXCEXPORT }_{t}+\beta_{6} \text { LINF }_{t}+u_{t}
\end{aligned}
$$$$
\text { TXCRESC }_{t}=\beta_{0}+\beta_{1} \text { TXCRESC }_{t-1}+\beta_{2} \text { SUBVALOR }_{t}+\beta_{3} \text { SOBREVALOR }_{t}
$$$$
+\beta_{4} \text { TXINVEST }_{t}+\beta_{5} \text { VOLAT }_{t}+\beta_{6} \text { TXCEXPORT }_{t}+\beta_{7} \text { LINF }_{t}+u_{t}
$$

Sendo:

TXCRESC = Taxa de crescimento do PIB $(\%)$, preços de mercado, índice encadeado, dessazonalizado (média $1995=100$ ). Fonte: IBGE (2013).

TXCRESCt-1 = Taxa de crescimento do PIB (\%) defasada em um período, preços de mercado, índice encadeado, dessazonalizado (média $1995=100$ ). Fonte: IBGE (2013).

DESALTOTAL = Medida de desalinhamento cambial total (\%). Fonte: Elaboração dos autores.

\footnotetext{
${ }^{7}$ No caso dos modelos estimados por GMM com a variável dependente defasada (Tabelas 3 e 5), a inclusão da taxa de crescimento defasada como instrumento, se inicia em $\mathrm{t}-2$. O número de defasagens dos instrumentos utilizados na estimação dos modelos GMM (Tabelas 3 e 5) foi 5 e tal escolha foi baseada na análise da Estatística J e sua probabilidade, onde a hipótese nula a ser testada é de que as condições sobreindentificadoras (conjunto de instrumentos) são válidas, e neste sentido a estimação dos modelos GMM com um menor número de defasagens (foi testado para 4, 3, 2 e 1 defasagens) fornecia resultados insatisfatórios, ou seja, Estatísticas J com probabilidade menor que 5\% (e em alguns casos menor que $10 \%$ ), o que invalidaria a utilização destes conjuntos de instrumentos.
} 
TXINVEST $=$ Taxa de investimento, formação bruta de capital fixo (\% do PIB). Fonte: Ipeadata (2013).

VOLAT $=$ Duas medidas de volatilidade cambial, VOLATCOND e DESVPADMOV. Fonte: Elaboração dos autores.

TXCEXPORT $=$ Taxa de crescimento das exportações (\%). Fonte: Ipeadata (2013).

LINF $=\log (1+$ inflação medida pelo IPCA) ( $\%$ ao ano). Fonte: Ipeadata (2013).

SUBVALOR = obtida a partir da construção de uma variável DUTOT = 1 para desalinhamento total positivo e 0 para negativo e depois multiplicado o DESALTOTAL por DUTOT. É uma medida de subvalorização cambial.

SOBREVALOR = obtida a partir da construção de uma variável DUTOT = 1 para desalinhamento positivo e 0 para negativo e depois multiplicando o DESALTOT por 1 - DUTOT. É uma medida de sobrevalorização cambial.

A escolha das variáveis para a estimação das equações de crescimento está associada aos diversos trabalhos da literatura de crescimento econômico desde as abordagens associadas ao modelo de Solow, como é o caso da inclusão da taxa de investimento (TXINVEST) e uma proxy para estabilidade macroeconômica com a inclusão da inflação (LINF). Além disso, tendo por base os argumentos propostos pela literatura de Export Led Growth, foi incluída a taxa de crescimento das exportações (TXCEXPORT). As demais variáveis são a taxa de crescimento defasada para introduzir um elemento dinâmico à equação estimada, e as variáveis de interesse deste trabalho que estão representadas pelas duas medidas de volatilidade cambial (VOLATCOND e DESVPADMOV), o desalinhamento cambial (DESALTOTAL) e as variáveis que indicam se a taxa de câmbio está subvalorizada ou sobrevalorizada (SUBVALOR e SOBREVALOR). Quanto às correlações esperadas os coeficientes seriam positivos para a taxa de investimento, a taxa de crescimento das exportações e o desalinhamento cambial e negativos para a inflação e a volatilidade cambial.

\section{APRESENTAÇÃO DOS RESULTADOS}

A Tabela 1 sistematiza os testes de estacionariedade das séries de tempo para que possam ser utilizadas na equação de crescimento apenas as séries estacionárias. Os resultados dos testes ADF, Phillips-Perron (PP), KPSS e DF-GLS indicam que as variáveis a serem consideradas como estacionárias são TXCRESC, LINF, VOLATCOND, DESVPADMOV, TXCEXPORT e DESALTOTAL, enquanto LREER e TXINVEST são não estacionárias e serão utilizadas em primeira diferença ${ }^{8}$.

\footnotetext{
${ }^{8}$ A variação do $\log$ da taxa de câmbio real efetiva (DLREER) não foi utilizada diretamente como
} 
Tabela 1: Testes de Estacionariedade - ADF, PP, KPSS

e DF-GLS - 1995 T1 a 2011 T4

\begin{tabular}{|c|c|c|c|c|c|c|c|c|}
\hline Variáveis / Testes & ADF & $\begin{array}{l}\text { Ordem de } \\
\text { Integração }\end{array}$ & PP & $\begin{array}{l}\text { Ordem de } \\
\text { Integração }\end{array}$ & KPSS & $\begin{array}{l}\text { Ordem de } \\
\text { Integração }\end{array}$ & DF-GLS & $\begin{array}{l}\text { Ordem de } \\
\text { Integração }\end{array}$ \\
\hline TXCRESC & $\begin{array}{l}-7,104 \\
* * * b\end{array}$ & $\mathrm{I}(0)$ & $\begin{array}{l}-7,085 \\
* * * b\end{array}$ & $I(0)$ & $0,286 b$ & $I(0)$ & $\begin{array}{l}-7,348 \\
* * * a\end{array}$ & $I(0)$ \\
\hline LREER & $-1,743 a$ & $I(1)$ & $-1,640 \mathrm{a}$ & I(1) & $\begin{array}{l}0,255 \\
* * a\end{array}$ & $I(1)$ & $-1,013 a$ & $I(1)$ \\
\hline LINF & $\begin{array}{l}-3,009 \\
* * * b\end{array}$ & $I(0)$ & $\begin{array}{l}-5,324 \\
* * * b\end{array}$ & $I(0)$ & $0,083 a$ & $I(0)$ & $-1,903 * b$ & $\mathrm{I}(0)$ \\
\hline TXINVEST & $-1,867$ a & $I(1)$ & $\begin{array}{l}-4,713 \\
* * * a\end{array}$ & $I(0)$ & $\begin{array}{l}0,278 \\
* * * a\end{array}$ & $I(1)$ & $-1,193 a$ & $I(1)$ \\
\hline VOLATCOND & $-2,545 a$ & $\mathrm{I}(1)$ & $\begin{array}{c}-2,867 \\
* * b\end{array}$ & I(0) & $0,104 b$ & $I(0)$ & $\begin{array}{l}-2,262 \\
* * * b\end{array}$ & $I(0)$ \\
\hline DESVPADMOV & $\begin{array}{c}-3,246 \\
* * b\end{array}$ & $\mathrm{I}(0)$ & $\begin{array}{l}-3,451 \\
* * b\end{array}$ & $\mathrm{I}(0)$ & $0,080 \mathrm{~b}$ & $\mathrm{I}(0)$ & $\begin{array}{l}-2,896 \\
* * * b\end{array}$ & $\mathrm{I}(0)$ \\
\hline TXCEXPORT & $\begin{array}{c}-3,293 \\
* * b\end{array}$ & $I(0)$ & $\begin{array}{l}-11,376 \\
* * * b\end{array}$ & $I(0)$ & $0,307 \mathrm{~b}$ & $I(0)$ & $-2,608 a$ & $\mathrm{I}(1)$ \\
\hline DESALTOTAL & $\begin{array}{l}-2,652 \\
* * * \mathrm{C}\end{array}$ & $I(0)$ & $\begin{array}{c}-2,287 \\
{ }^{*}{ }^{*} \mathrm{C}\end{array}$ & $\mathrm{I}(0)$ & $\begin{array}{l}0,164 \\
*{ }^{*} \mathrm{a}\end{array}$ & $\mathrm{I}(1)$ & $\begin{array}{c}-3,257 \\
* * a\end{array}$ & $\mathrm{I}(0)$ \\
\hline
\end{tabular}

Notas: ADF, PP e DF-GLS estatística t e KPSS estatística LM. Hipótese nula para ADF, PP e DF-GLS = Série possui Raiz Unitária. Hipótese nula para KPSS $=$ Série é Estacionária. ${ }^{*},{ }^{*} e^{* * *}$ indicam rejeição da hipótese nula a $10 \%$, $5 \%$ e $1 \%$, respectivamente. I(1) indica integrada de ordem um e não estacionária; I(0) indica integrada de ordem zero e estacionária. $a$ = indica com constante e tendência. $b=$ indica com constante e sem tendência. $c=$ sem constante e tendência.

A estimação das equações (3) e (4) para a taxa de crescimento da economia brasileira no período de 1995T1 a 2011T4 foi feita por MQO e GMM.

Os resultados das estimações por MQO do modelo de crescimento, com a variável dependente defasada, sistematizados na Tabela 2, indicam que os coeficientes associados à variável DESALTOTAL são positivos e estatisticamente significativos em todos os modelos estimados, com coeficientes variando de 0,021 a 0,041, sugerindo que um aumento no valor de DESALTOTAL (diminuição da sobrevalorização cambial/aumento da subvalorização cambial) de $10 \%$ tem um impacto positivo na taxa de crescimento da economia brasileira da ordem de $0,21 \%$ a $0,41 \%$, e um impacto negativo da mesma magnitude no caso de uma diminuição no valor de DESALTOTAL (aumento da sobrevalorização cambial/diminuição da subvalorização cambial).

variável de controle nas estimações MQO e GMM, mas foi utilizada como instrumento com valores correntes e com 5 defasagens nas estimações dos diversos modelos de crescimento estimado por GMM. 
Tabela 2: Modelo de Crescimento - Estimação MQO -

Desalinhamento Cambial e Volatilidade Cambial

\begin{tabular}{|c|c|c|c|c|c|c|c|}
\hline Modelos & 1 & 2 & 3 & 4 & $5 \#$ & $6 \#$ & $7 \#$ \\
\hline TXCRESC $(-1)$ & 0,050 & $-0,113$ & $-0,117$ & $-0,126$ & 0,016 & $-0,000$ & $-0,000$ \\
\hline Erro-padrão & $(0,121)$ & $(0,124)$ & $(0,122)$ & $(0,123)$ & $(0,157)$ & $(0,144)$ & $(0,145)$ \\
\hline DESALTOTAL & 0,028 & 0,041 & 0,037 & 0,038 & 0,024 & 0,021 & 0,021 \\
\hline Erro-padrão & $(0,010) * * *$ & $(0,011) * * *$ & $(0,011) * * *$ & $(0,011) * * *$ & $(0,007) * *$ & $(0,008) * *$ & $(0,008) * *$ \\
\hline DTXINVEST & & 0,191 & 0,191 & 0,188 & 0,232 & 0,228 & 0,228 \\
\hline Erro-padrão & & $(0,126)$ & $(0,124)$ & $(0,124)$ & $(0,152)$ & $(0,130) *$ & $(0,132) *$ \\
\hline DESVPADMOV & & $-9,924$ & $-9,075$ & $-9,989$ & & & \\
\hline Erro-padrão & & $(3,764) * * *$ & $(3,725) * *$ & $(3,898) * *$ & & & \\
\hline VOLATCOND & & & & & $-10,358$ & $-9,540$ & $-9,519$ \\
\hline Erro-padrão & & & & & $(10,814)$ & $(10,050)$ & $(10,066)$ \\
\hline TXCEXPORT & & & 0,017 & 0,018 & & 0,019 & 0,019 \\
\hline Erro-padrão & & & $(0,009) *$ & $(0,009) *$ & & $(0,010) *$ & $(0,011) *$ \\
\hline LINF & & & & 0,040 & & & 0,002 \\
\hline Erro-padrão & & & & $(0,049)$ & & & $(0,038)$ \\
\hline $\mathrm{R} 2$ & 0,117 & 0,249 & 0,289 & 0,297 & 0,174 & 0,227 & 0,227 \\
\hline F-Stat & 4,046 & 4,909 & 4,723 & 4,025 & 3,113 & 3,419 & 2,800 \\
\hline Prob. F-Stat & 0,022 & 0,001 & 0,001 & 0,001 & 0,021 & 0,008 & 0,018 \\
\hline Teste BG - Prob. & 0,326 & 0,619 & 0,350 & 0,316 & 0,037 & 0,049 & 0,049 \\
\hline Teste White - Prob. & 0,787 & 0,259 & 0,825 & 0,918 & 0,243 & 0,586 & 0,806 \\
\hline
\end{tabular}

Notas: ${ }^{*},{ }^{*} e^{* * *}$ indicam significância estatística a 10\%, 5\% e 1\%. Não foram reportados os coeficientes da constante. \# Modelo Estimado com a Correção de Newey-West. Teste BG de Autocorrelação - Hipótese Nula de Ausência de Autocorrelação. Teste White de Homocedasticidade - Hipótese Nula de Homocedasticidade.

Ao analisar os resultados das estimações dos coeficientes das duas proxies de volatilidade cambial (DESVPADMOV e VOLATCOND) percebe-se que apenas DESVPADMOV é estatisticamente significativa, embora ambas as medidas possuam coeficientes com sinais negativos, indicando que um aumento (diminuição) na volatilidade da taxa de câmbio real efetiva diminui (aumenta) a taxa de crescimento. Quanto à variação da taxa de investimento (DTXINVEST), os coeficientes estimados são positivos e só se mostraram significativos em dois dos três modelos onde foi utilizada a proxy de VOLATCOND (modelos 6 e 7), porém em todos os casos a significância só foi obtida ao nível de $10 \%$.

A estimação dos coeficientes da taxa de crescimento das exportações (TXCEXPORT) revela que os coeficientes foram todos positivos e estatisticamente significativos (a 10\%), enquanto no caso da taxa de inflação (LINF) as estimativas revelam que os coeficientes são positivos, mas não significativos. Os testes de ausência de autocorrelação indicaram rejeição da hipótese nula nos modelos 5, 6 e 7, e para estes modelos os erros-padrão foram corrigidos pelo método de Newey-West. Além disso, os modelos estimados não têm problema de heterocedasticidade. 
Tabela 3: Modelo de Crescimento - Estimação GMM -

Desalinhamento Cambial e Volatilidade Cambial

\begin{tabular}{|c|c|c|c|c|c|c|c|}
\hline Modelos & 1 & 2 & 3 & 4 & 5 & 6 & 7 \\
\hline TXCRESC (t-1) & 0,354 & 0,103 & 0,126 & 0,089 & 0,294 & 0,266 & 0,245 \\
\hline Erro-padrão & $\begin{array}{c}(0,077) \\
* * *\end{array}$ & $(0,091)$ & $(0,065) *$ & $(0,038) * *$ & $(0,047) * * *$ & $(0,041) * * *$ & $\begin{array}{l}(0,032) \\
* * *\end{array}$ \\
\hline DESALTOTAL & 0,029 & 0,037 & 0,030 & 0,032 & 0,027 & 0,018 & 0,017 \\
\hline Erro-padrão & $\begin{array}{c}(0,006) \\
* * *\end{array}$ & $(0,005) * * *$ & $(0,004) * * *$ & $(0,003) * * *$ & $(0,004) * * *$ & $(0,003) * * *$ & $\begin{array}{c}(0,003) \\
* * *\end{array}$ \\
\hline DTXINVEST & & 0,075 & 0,105 & 0,131 & 0,078 & 0,133 & 0,150 \\
\hline Erro-padrão & & $(0,045) *$ & $(0,046) * *$ & $(0,037) * * *$ & $(0,040) *$ & $(0,031) * * *$ & $\begin{array}{c}(0,029) \\
* * *\end{array}$ \\
\hline DESVPADMOV & & $-6,139$ & $-5,212$ & $-8,443$ & & & \\
\hline Erro-padrão & & $(2,371) * *$ & $(1,561) * * *$ & $(1,240) * * *$ & & & \\
\hline VOLATCOND & & & & & $-1,866$ & $-10,814$ & $-9,852$ \\
\hline Erro-padrão & & & & & $(4,661)$ & $(3,491) * * *$ & $\begin{array}{l}(2,275) \\
* * *\end{array}$ \\
\hline TXCEXPORT & & & 0,015 & 0,022 & & 0,014 & 0,018 \\
\hline Erro-padrão & & & $(0,003) * * *$ & $(0,002) * * *$ & & $(0,004) * * *$ & $\begin{array}{l}(0,004) \\
* * *\end{array}$ \\
\hline LINF & & & & 0,086 & & & 0,023 \\
\hline Erro-padrão & & & & $(0,012) * * *$ & & & $(0,016)$ \\
\hline R2 & 0,146 & 0,270 & 0,327 & 0,357 & 0,183 & 0,266 & 0,284 \\
\hline J-Statistics & 8,701 & 11,384 & 13,846 & 14,227 & 11,733 & 13,671 & 13,773 \\
\hline Prob. J. Statistics & 0,795 & 0,954 & 0,964 & 0,989 & 0,946 & 0,967 & 0,992 \\
\hline $\begin{array}{c}\text { Teste de } \\
\text { Endogeneidade - Prob. }\end{array}$ & 0,740 & 0,925 & 0,990 & 0,999 & 0,946 & 0,971 & 0,995 \\
\hline
\end{tabular}

Notas: ${ }^{*},{ }^{*} e^{* * *}$ indicam significância estatística a 10\%, 5\% e 1\%. Teste de Endogeneidade - Difference in J-stats - Hipótese Nula: Regressores Exógenos. Não foram reportados os coeficientes da constante.

As estimações por GMM do modelo de crescimento sistematizadas na Tabela 3 para o modelo com a variável dependente defasada mostram que os coeficientes associados à DESALTOTAL são todos positivos e estatisticamente significativos, variando de 0,017 a 0,037, indicando que aumento no valor de DESALTOTAL (diminuição da sobrevalorização cambial/aumento da subvalorização cambial) de $10 \%$ tem um impacto positivo na taxa de crescimento da economia brasileira da ordem de $0,17 \%$ a $0,37 \%$, e um impacto negativo da mesma magnitude no caso de uma diminuição no valor de DESALTOTAL (aumento da sobrevalorização cambial/diminuição da subvalorização cambial).

Os resultados das estimações dos coeficientes para as duas proxies de volatilidade cambial (DESVPADMOV e VOLATCOND) revela que os mesmos são negativos em ambos os casos, e estatisticamente significativos para todos os modelos com exceção do modelo 5, que inclui VOLATCOND. O sinal negativo as- 
sociado às duas proxies para a volatilidade cambial indica que um aumento (diminuição) na volatilidade da taxa de câmbio real efetiva diminui (aumenta) a taxa de crescimento.

As estimativas dos coeficientes para a variação da taxa de investimento (DTXINVEST) mostram que os coeficientes são todos positivos e estatisticamente significativos. Os coeficientes estimados para a taxa de crescimento das exportações (TXCEXPORT) e a taxa de inflação (LINF) indicam que ambos possuem coeficientes positivos, sendo que no caso de TXCEXPORT os mesmos são significativos em todos os modelos estimados, enquanto para LINF apenas no modelo 4, onde se utiliza a proxy de volatilidade cambial DESVPADMOV foi verificada significância estatística. A estatística $\mathrm{J}$ indica que os instrumentos são válidos enquanto o teste de endogeneidade revela que o conjunto dos regressores é exógeno.

Os resultados das estimações por MQO (Tabela 4) e por GMM (Tabela 5) analisam o papel da subvalorização e sobrevalorização da taxa de câmbio real efetiva sobre o crescimento econômico. Ademais, os resultados apresentados permitem avaliar o papel da volatilidade cambial sobre o crescimento econômico.

A estimação do modelo de crescimento por MQO revela que os coeficientes estimados para SUBVALOR e SOBREVALOR são todos positivos, indicando que uma taxa de câmbio real efetiva subvalorizada (sobrevalorizada) estimula (desestimula) o crescimento econômico. Os coeficientes estimados para SUBVALOR nos modelos 2, 3 e 4 são estaticamente significativos enquanto para SOBREVALOR a significância foi encontrada apenas nos modelos 2 e 3, sendo que em ambos os casos de significância para SUBVALOR e SOBREVALOR estes modelos incluem a proxy de volatilidade cambial medida por DESVPADMOV. Analisando apenas os coeficientes estatisticamente significativos, a magnitude para SUBVALOR varia de 0,037 a 0,044 indicando que uma subvalorização cambial da ordem de $10 \%$ tem um impacto positivo sobre o crescimento econômico da ordem de 3,7\% a 4,4\%. Já no caso dos coeficientes para SOBREVALOR, cuja magnitude varia entre 0,037 e 0,036 , uma sobrevalorização cambial de $10 \%$ tem um impacto negativo sobre o crescimento econômico de 3,6\%.

A análise das estimações considerando as duas proxies de volatilidade cambial (DESVPADMOV e VOLATCOND) revela que apenas DESVPADMOV é estatisticamente significativa e com coeficiente estimado negativo, indicando que um aumento (diminuição) na volatilidade da taxa de câmbio real efetiva desestimula (estimula) o crescimento econômico da economia brasileira.

Analisando as demais variáveis do modelo percebe-se que a variação da taxa de investimento (DTXINVEST) tem coeficientes estimados positivos mas significativos apenas nos modelos 6 e 7 (a 10\%), a taxa de crescimento das exportações (TXCEXPORT) tem coeficientes estimados positivos e é significativa em todos os modelos. Por fim, a taxa de inflação (LINF) não se mostrou significativa nos dois modelos estimados. 
Tabela 4: Modelo de Crescimento - Estimação MQO -

Subvalorização, Sobrevalorização e Volatilidade Cambial

\begin{tabular}{|c|c|c|c|c|c|c|c|}
\hline Modelos & 1 & 2 & 3 & 4 & $5 \#$ & $6 \#$ & $7 \#$ \\
\hline TXCRESC (-1) & 0,052 & $-0,109$ & $-0,117$ & $-0,124$ & 0,018 & $-0,004$ & $-0,004$ \\
\hline Erro-padrão & $(0,123)$ & $(0,126)$ & $(0,124)$ & $(0,125)$ & $(0,174)$ & $(0,160)$ & $(0,165)$ \\
\hline SUBVALOR & 0,030 & 0,044 & 0,037 & 0,042 & 0,025 & 0,019 & 0,019 \\
\hline Erro-padrão & $(0,018)$ & $(0,018) * *$ & $(0,018) *$ & $(0,019) * *$ & $(0,015)$ & $(0,015)$ & $(0,017)$ \\
\hline SOBREVALOR & 0,025 & 0,037 & 0,036 & 0,034 & 0,023 & 0,024 & 0,024 \\
\hline Erro-padrão & $(0,022)$ & $(0,021) *$ & $(0,021) *$ & $(0,021)$ & $(0,024)$ & $(0,022)$ & $(0,024)$ \\
\hline DTXINVEST & & 0,187 & 0,191 & 0,185 & 0,231 & 0,230 & 0,230 \\
\hline Erro-padrão & & $(0,128)$ & $(0,126)$ & $(0,127)$ & $(0,147)$ & $(0,129) *$ & $(0,130) *$ \\
\hline DESVPADMOV & & $-10,015$ & $-9,076$ & $-10,164$ & & & \\
\hline Erro-padrão & & $(3,817) * *$ & $(3,786) * *$ & $(4,010) * *$ & & & \\
\hline VOLATCOND & & & & & $-10,471$ & $-9,302$ & $-9,300$ \\
\hline Erro-padrão & & & & & $(11,864)$ & $(10,983)$ & $(11,037)$ \\
\hline TXCEXPORT & & & 0,017 & 0,017 & & 0,020 & 0,020 \\
\hline Erro-padrão & & & $(0,009) *$ & $(0,009) *$ & & $(0,010) *$ & $(0,011) *$ \\
\hline LINF & & & & 0,043 & & & 0,000 \\
\hline Erro-padrão & & & & $(0,051)$ & & & $(0,044)$ \\
\hline R2 & 0,117 & 0,250 & 0,289 & 0,298 & 0,174 & 0,227 & 0,227 \\
\hline F-Stat & 2,659 & 3,873 & 3,868 & 3,40 & 2,450 & 2,805 & 2,362 \\
\hline Prob. F-Stat & 0,056 & 0,004 & 0,002 & 0,004 & 0,044 & 0,018 & 0,034 \\
\hline Teste BG - Prob. & 0,302 & 0,594 & 0,349 & 0,282 & 0,028 & 0,056 & 0,056 \\
\hline Teste White - Prob. & 0,575 & 0,187 & 0,665 & 0,532 & 0,299 & 0,554 & 0,398 \\
\hline
\end{tabular}

Notas: ${ }^{*},{ }^{*} e^{* * *}$ indicam significância estatística a 10\%, 5\% e 1\%. Não foram reportados os coeficientes da constante. \# Modelo Estimado com a Correção de Newey-West. Teste BG de Autocorrelação - Hipótese Nula de Ausência de Autocorrelação. Teste White de Homocedasticidade - Hipótese Nula de Homocedasticidade.

Os testes de ausência de autocorrelação e de heterocedasticidade revelam que apenas os modelos 5, 6 e 7 indicam a presença de autocorrelação.

A estimação do modelo de crescimento por GMM (Tabela 5) revela que os coeficientes estimados para SUBVALOR e SOBREVALOR são todos positivos, indicando que uma taxa de câmbio real efetiva subvalorizada (sobrevalorizada) estimula (desestimula) o crescimento econômico. Os coeficientes estimados para SUBVALOR são significativos em todos os modelos estimados, enquanto no caso de SOBREVALOR a significância foi encontrada apenas nos modelos 1 a 4, sendo que os modelos 2, 3 e 4 incluem a proxy de volatilidade cambial medida por DESVPADMOV. Analisando apenas os coeficientes estatisticamente significativos, a magnitude para SUBVALOR varia de 0,029 a 0,056 indicando que uma taxa de câmbio real efetiva subvalorizada em $10 \%$ tem um impacto positivo sobre o crescimento econômico da ordem de 2,9\% a 5,6\%. Já no caso dos coeficientes para SOBREVALOR, cuja magnitude varia entre 0,011 e 0,021, indica que uma taxa de 
câmbio real efetiva sobrevalorizada em $10 \%$ tem um impacto negativo sobre o crescimento econômico da ordem de $1,1 \%$ a $2,1 \%$.

Tabela 5: Modelo de Crescimento - Estimação GMM -

Subvalorização, Sobrevalorização e Volatilidade Cambial

\begin{tabular}{|c|c|c|c|c|c|c|c|}
\hline Modelos & 1 & 2 & 3 & 4 & 5 & 6 & 7 \\
\hline TXCRESC (t-1) & 0,399 & 0,132 & 0,087 & 0,071 & 0,345 & 0,270 & 0,251 \\
\hline Erro-padrão & $(0,046) * * *$ & $(0,081)$ & $(0,063)$ & $(0,028) * *$ & $(0,043) * * *$ & $(0,046) * * *$ & $\begin{array}{c}(0,029) \\
* * *\end{array}$ \\
\hline SUBVALOR & 0,033 & 0,051 & 0,044 & 0,056 & 0,035 & 0,029 & 0,029 \\
\hline Erro-padrão & $(0,008) * * *$ & $(0,009) * * *$ & $(0,006) * * *$ & $(0,005) * * *$ & $(0,005) * * *$ & $(0,005) * * *$ & $\begin{array}{c}(0,002) \\
* * *\end{array}$ \\
\hline SOBREVALOR & 0,021 & 0,019 & 0,020 & 0,011 & 0,008 & 0,004 & 0,002 \\
\hline Erro-padrão & $(0,010) * *$ & $(0,007) * *$ & $(0,007) * * *$ & $(0,006) *$ & $(0,011)$ & $(0,008)$ & $(0,005)$ \\
\hline DTXINVEST & & 0,054 & 0,088 & 0,089 & 0,067 & 0,106 & 0,123 \\
\hline Erro-padrão & & $(0,039)$ & $(0,033) * *$ & $(0,020) * * *$ & $(0,031) * *$ & $(0,028) * * *$ & $\begin{array}{c}(0,028) \\
* * *\end{array}$ \\
\hline DESVPADMOV & & $-6,641$ & $-7,090$ & $-10,819$ & & & \\
\hline Erro-padrão & & $(2,462) * * *$ & $(1,580) * * *$ & $(1,284) * * *$ & & & \\
\hline VOLATCOND & & & & & $-8,403$ & $-13,803$ & $-12,254$ \\
\hline Erro-padrão & & & & & $(5,346)$ & $(3,293) * * *$ & $\begin{array}{c}(1,824) \\
* * *\end{array}$ \\
\hline TXCEXPORT & & & 0,016 & 0,020 & & 0,015 & 0,018 \\
\hline Erro-padrão & & & $(0,002) * * *$ & $(0,002) * * *$ & & $(0,002) * * *$ & $\begin{array}{c}(0,002) \\
* * *\end{array}$ \\
\hline LINF & & & & 0,109 & & & 0,037 \\
\hline Erro-padrão & & & & $(0,012) * * *$ & & & $\begin{array}{c}(0,013) \\
* * *\end{array}$ \\
\hline $\mathrm{R} 2$ & 0,138 & 0,278 & 0,340 & 0,367 & 0,196 & 0,274 & 0,290 \\
\hline J-Statistics & 10,377 & 13,337 & 14,144 & 14,231 & 13,630 & 14,124 & 14,135 \\
\hline Prob. J. Statistics & 0,887 & 0,972 & 0,990 & 0,998 & 0,967 & 0,990 & 0,998 \\
\hline $\begin{array}{l}\text { Teste de Endogeneida- } \\
\text { de - Prob. }\end{array}$ & 0,610 & 0,964 & 0,997 & 1,000 & 0,982 & 0,997 & 0,999 \\
\hline
\end{tabular}

Notas: ${ }^{*},{ }^{*} e^{* * *}$ indicam significância estatística a 10\%, 5\% e 1\%. Teste de Endogeneidade - Difference in J-stats - Hipótese Nula: Regressores Exógenos. Não foram reportados os coeficientes da constante.

A análise das estimações considerando as duas proxies de volatilidade cambial (DESVPADMOV e VOLATCOND) revela que os coeficientes estimados são estatisticamente significativos, com exceção do modelo 5 para VOLATCOND. Todos os coeficientes estimados são negativos, indicando que um aumento (diminuição) na volatilidade da taxa de câmbio real efetiva desestimula (estimula) o crescimento econômico da economia brasileira.

Analisando as demais variáveis do modelo percebe-se que a variação da taxa de investimento (DTXINVEST) tem coeficientes estimados positivos sendo que a significância estatística foi observada em todos os modelos com exceção do mode- 
lo 2. A taxa de crescimento das exportações (TXCEXPORT) tem coeficientes estimados positivos e estatisticamente significativos em todos os modelos. Por fim, os coeficientes associados à taxa de inflação (LINF) nos modelos 4 e 7 são positivos e estatisticamente significativos.

A estatística $\mathrm{J}$ indica que os instrumentos são válidos enquanto o teste de endogeneidade revela que o conjunto dos regressores é exógeno.

\section{CONSIDERAÇÕES FINAIS}

Este trabalho contribui para a literatura empírica sobre o crescimento econômico da economia brasileira por apresentar evidências sistemáticas acerca das relações entre: i) desalinhamento cambial e crescimento econômico; ii) volatilidade cambial e crescimento econômico. Para tanto: i) constrói a série de taxa de câmbio real efetiva para o período 1994T3-2011T4 considerando os vinte principais parceiros comerciais; ii) calcula uma medida de desalinhamento cambial, uma medida de subvalorização cambial, uma medida de sobrevalorização cambial e duas medidas de volatilidade cambial. Por fim, estima-se equações de crescimento utilizando dados trimestrais para o período $1995 \mathrm{~T} 1$ a 2011T4, tendo como métodos de estimação MQO e GMM.

Os resultados das estimações das equações de crescimento econômico para a economia brasileira sugerem: i) diminuição da sobrevalorização cambial/aumento da subvalorização cambial (aumento da sobrevalorização cambial/diminuição da subvalorização cambial) estimula (desestimula) o crescimento econômico; subvalorização (sobrevalorização) cambial estimula (desestimula) o crescimento econômico; maior (menor) volatilidade cambial desestimula (estimula) o crescimento econômico. Ou seja, a lição a ser assimilada das evidências apresentadas para a economia brasileira é a de que uma taxa de câmbio real efetiva em um nível competitivo e com baixa volatilidade estimula o crescimento econômico.

Por fim, os resultados apresentados para a experiência brasileira durante o período 1995T1-2011T4 corroboram o argumento de Eichengreen (2008), segundo o qual a combinação de taxa de câmbio real efetiva em nível competitivo e baixa volatilidade da taxa de câmbio real efetiva é favorável às economias em desenvolvimento e emergentes, onde um setor exportador dinâmico é normalmente parte importante do processo para alcançar taxas de crescimento econômico elevadas e sustentadas. Como recomendação de política, sugere-se a manutenção da taxa de câmbio real efetiva em nível competitivo e com baixa volatilidade. Conforme Eichengreen (2008, p. 4): “Keeping it at appropriate levels and avoiding excessive volatility enable a country to exploit its capacity for growth and development". 


\section{REFERÊNCIAS BIBLIOGRÁFICAS}

AGHION, P.; BACCHETTA, P.; RANCIERE, R.; ROGOFF, K. (2009) "Exchange rate volatility and productivity growth: The role of financial development". Journal of Monetary Economics, Vol. 56, no 4, p. 494-513.

AGUIRRE, A.; CALDERÓN, C. (2005) "Real exchange rate misalignments and economic performance". Central Bank of Chile, Working Papers n ${ }^{\circ} 315$.

BANCO CENTRAL DO CHILE (2013) Acesso disponível em: http://www.bcentral.cl/index.asp

BELKE, A.; KAAS, L. (2004) "Exchange rate movements and employment growth: An OCA assessment of the CEE economies". Empirica, Vol. 31, n 2-3, p. 247-280.

BERG, A.; MIAO, Y. (2010) "The real exchange rate and growth revisited: The Washington Consensus strikes back?" International Monetary Fund, IMF Working Paper $n^{\circ} 58$.

BEVERIDGE, S.; NELSON, C. R. (1981) "A new approach to decomposition of economic time series into permanent and transitory components, with particular attention to the measurement of business cycles”. Journal of Monetary Economics, Vol. 7, nº 2, p. 151-174.

BLEANEY, M.; GREENAWAY, D. (2001) "The impact of terms of trade and real exchange rate volatility on investment and growth in Sub-Saharan Africa. Journal of Development Economics, Vol. $65, \mathrm{n}^{\circ} 2,491-500$.

BOSWORTH, B.; COLLINS, S.; CHEN, Y. (1996) “Accounting for differences in economic growth". In KOHSAKA, A.; OHNO, K. (orgs.). Structural Adjustment and Economic Reform: East Asia, Latin America, and Central and Eastern Europe. Tokyo: Institute of Developing Economies.

BREDIN, D.; FOUNTAS, S.; MURPHY, E. (2003) "An empirical analysis of short run and long run irish export functions: Does exchange rate volatility matter? International Review of Applied Economics, Vol. 17, n' 2, p. 193-208.

BRESSER-PEREIRA, L. C. (2008) "The Dutch disease and its neutralization: a ricardian approach". Revista de Economia Política, Vol. 28, N.1.

BRESSER-PEREIRA, L. C. (2009a) Globalização e Competição. Rio de Janeiro: Elsevier-Campus.

BRESSER-PEREIRA, L. C. (2009b) "A tendência à sobreapreciação da taxa de câmbio". Econômica, Vol. 11, $\mathrm{n}^{\mathrm{o}}$ 1, p.7-30.

BRESSER-PEREIRA, L. C. (2012) "A taxa de câmbio no centro da teoria do desenvolvimento". Estudos Avançados, Vol. 26, nº 75, p. 7-28.

BRESSER-PEREIRA, L. C.; GALA, P. (2010) “Macroeconomia estruturalista do desenvolvimento". Revista de Economia Política, vol. 30, n 4, p. 663-686.

CABALLERO, R. J.; CORBO, I. (1989) "The effect of real exchange rate uncertainty on exports: Empirical evidence". The World Bank Economic Review, Vol. 3, n 2, p. 263-78.

CAMPA, J.; GOLDBERG, L. S. (1995) "Investment in manufacturing, exchange rates and external exposure". Journal of International Economics, Vol. 38, n 3-4, p. 297-320, 1995.

CHINA STATISTICAL YEARBOOK (2013) National Bureau of Statistics of China. Disponível em: http://www.stats.gov.cn/english/statisticaldata/Quarterlydata/

CLARK, P.; MACDONALD, R. (1999) "Exchange rates and economic fundamentals: a methodological comparison of BEERs and FEERs. In: MACDONALD, R.; STEIN, J. L (orgs.). Equilibrium Exchange Rates. Amsterdam: Kluwer.

CRAGG, J. G. (1983) "More efficient estimation in the presence of heteroscedasticity of unknown form. Econometrica, Vol. 51, n ${ }^{\circ}$ 3, p. 751-763.

CUSHMAN, D. O. (1986) "Has exchange risk depressed international trade? The impact of third country exchange risk." Journal of International Money and Finance, Vol. 5, n ${ }^{\circ}$ 3, p. 361-379.

DARBY, J.; HALLETT, A. H.; IRELAND, J.; PISCITELLI, L. (1999) "The impact of exchange rate uncertainty on the level of investment”. The Economic Journal, Vol. 109, n 454, p. 55-67.

DOLLAR, D. (1992) “Outward oriented developing countries really do grow more rapidly". Economic Development and Cultural Change, Vol. 40, ${ }^{\circ} 3,523-554$.

DOYLE, E. (2001) “Exchange rate volatility and Irish-UK trade, 1979-1992". Applied Economics, Vol. $33, \mathrm{n}^{\circ} 2$, p. 249-65. 
EDWARDS, S. (1989) “Exchange rate misalignment in developing countries". The World Bank Research Observer, Vol. 4, n 1, p. 3-21.

EDWARDS, S. (1998) “Openness, productivity, and growth: what do we really know?" Economic Journal, Vol. 108, n ${ }^{\circ}$ 447, p.383-398.

EICHENGREEN, B. (2008) "The real exchange rate and economic growth". The World Bank, Commission on Growth and Development, Working Paper $n^{\circ} 4$.

ÉGERT, B.; HALPERN, L.; MACDONALD, R. (2006) "Equilibrium exchange rates in transition economies: Taking stock of the issues". Journal of Economic Surveys, Vol. 20, n 2.

ENDERS, W. (2004) Applied Econometric Time Series. 2. ed. Danvers: Willey.

EUROPEAN CENTRAL BANK (2013) Estatísticas do Banco Central Europeu. Disponível em: http:// www.ecb.int/stats/html/index.en.html

FARUQEE, H.; ISARD, P.; MASSON.A (1999) "Macroeconomic balance frameworkfor estimating equilibrium exchange rates”. In MACDONALD, R.; STEIN, J. L (orgs.). Equilibrium Exchange Rates. Amsterdam: Kluwer.

FRANKE, G. (1991) "Exchange rate volatility and international trading strategy". Journal of International Money and Finance, Vol.10, n 2, 292-307.

GALA, P.; LUCINDA, C. R. (2006) Exchange rate misalignment and growth: Old and new econometric evidence. Revista EconomiA, Vol. 7, nº 4, p.165-87.

GHOSH, A.; GULDE, A. M.; OSTRY, J. D.; WOLF, H. C. (1997) "Does the nominal exchange rate regime matter?” The National Bureau of Economic Research, NBER Working Paper n ${ }^{\circ} 5874,1997$.

GHURA, D.; GRENNES, T. J. (1993) “The real exchange rate and macroeconomic performance in Sub-Saharan Africa”. Journal of Development Economics, Vol. 42, n 1, p. 155-174.

HANSEN, L. P. (1982) "Large sample properties of generalized method of moments estimators". Econometrica, Vol. 50, no 4, p. 1029-1054.

HODRICK, R.; PRESCOTT, E. C. (1997) “Postwar U.S. business cycles: An empirical investigation". Journal of Money, Credit, and Banking, Vol. 29, $\mathrm{n}^{\circ}$ 1, 1-16.

IBGE. INSTITUTO BRASILEIRO DE GEOGRAFIA E ESTATÍSTICA, 2013.

INTERNATIONAL MONETARY FUND. Fiscal Monitor. IMF: Washington, 2012.

INTERNATIONAL MONETARY FUND. Direction of Trade Statistics. IMF: Washington, 2013.

INTERNATIONAL MONETARY FUND. International Financial Statistics. IMF: Washington, 2013.

INTERNATIONAL MONETARY FUND. World Economic Outlook Database. IMF: Washington, 2013.

INTERNATIONAL MONETARY FUND. Historical Public Debt Database. IMF: Washington, 2012.

IPEADATA. INSTITUTO DE PESQUISA ECONÔMICA APLICADA, 2013.

ISARD, P.; FARUQEE, H. (1998) Exchange rate assessment: extension of the macroeconomic balance approach. IMF Occasional Papers $\mathrm{n}^{\circ} 167$.

JOHANSEN, S. (1995) Likelihood-Based Inference in Cointegrated Vector Auto-Regressive Models. Oxford: Oxford University Press.

KRUEGER, A. O. (1983) Exchange Rate Determination. Cambridge: Cambridge University Press, 1983.

LANE, P. R.; MILESI-FERRETTI, G. M. (2007) “The external wealth of nations mark II: Revised and extended estimates of foreign assets and liabilities, 1970-2004. Journal of International Economics, Vol. 73, n 2, p.263-294. (versão atualizada do banco de dados)

MACDONALD, R. (2000) "Concepts to calculate equilibrium exchange rate: An overview. Economic Research Group of the Deustche Bundesbank Discussion Paper $n^{\circ} 3$.

MACDONALD, R.; DIAS, P. (2007) "Behavioural equilibrium exchange rate estimates and implied exchange rate adjustments for ten countries". University of Glasgow Discussion Paper $\mathrm{n}^{\circ} 10$.

MARCONI, N. (2012) "The industrial equilibrium exchange rate in Brazil: an estimation”. Revista de Economia Política, Vol. 32, n 4, p. 656-669.

MBAYE, S. (2013) “Currency undervaluation and growth: Is there a productivity channel?” International Economics, Vol. 133, p.8-28.

OECD STATISTICS (2013) Disponível em: http://stats.oecd.org/ 
OREIRO, J. L. e FEIJÓ, C. A. (2010) "Desindustrialização: conceituação, causas, efeitos e o caso brasileiro". Revista de Economia Política, Vol. 30, n 2 , p. 219-232.

PALMA, G. (2005) Quatro fontes de desindustrialização e um novo conceito de doença holandesa. Conferência de Industrialização, Desindustrialização e Desenvolvimento, Federação das Indústrias do Estado de São Paulo, Agosto, 2005.

PEREE, E.; STEINHERR, A. (1989) "Exchange rate uncertainty and foreign trade". European Economic Review, Vol. 33, n 6, p.1241-1264.

RAZIN, O.; COLLINS, S. M. (1997) "Real exchange rate misalignments and growth". The National Bureau of Economic Research, NBER Working Paper $n^{\circ} 6174$.

RODRIK, D. (2008) “The real exchange rate and economic growth". Brookings Papers on Economic Activity, $\mathrm{n}^{\circ}$ 2, p.365-412.

SERCU, P.; VANHULLE, C. (1992) "Exchange rate volatility, international trade, and the value of exporting firms". Journal of Banking and Finance, Vol. 16, $\mathrm{n}^{\circ}$ 1, p. 155-82.

SERVEN, L. (2002) "Real exchange rate uncertainty and private investment in developing countries". World Bank Policy Research Working Paper $n^{\circ} 2823$.

STEIN, J. L. (2002) “The equilibrium real exchange rate of the euro: an evaluation of research". CESifo Economic Studies, Vol.43, no 3, p. 349-381.

STEIN, J. L. (1995) "The fundamental determinants of the real exchange rate of the U.S.dollar relative to other G-7 countries". International Monetary Fund Working Paper n ${ }^{\circ} 81,1995$.

STEIN, J. L. (1994) "The natural real exchange rate of the US dollar and determinants ofcapital flows". In J. WILLIAMSON (org.). Estimating Equilibrium Exchange Rates. Washington D .C.: Institute for International Economics, p. 133-176.

VIAENE, J. M.; VRIES, C. G. (1992) “International Trade and Exchange Rate Volatility. European Economic Review, Vol. 36, n 6, p. 1311-1321.

VIEIRA, F. V.; HOLLAND, M.; SILVA, C. G.; BOTTECCHIA, L. C. (2013) "Growth and exchange rate volatility: a panel data analysis". Applied Economics, Vol. 45, n 26, p.3733-3741.

VIEIRA, F. V.; MACDONALD, R. (2012) "A panel data investigation of real exchange rate misalignment and growth". Estudos Econômicos, Vol. 42, n 3, p. 433-456.

WILLIAMSON, J. (1994) “Estimates of FEERs.” In J. WILLIAMSON (org.). Estimating Equilibrium Exchange Rates. Washington D .C.: Institute for International Economics, p. 177-244.

WILLIAMSON, J. (1983) “The exchange rate system". Institute for International Economics, Policy Analysis in International Economics $\mathrm{n}^{\circ} 5$.

WILLIAMSON, J. (1990) "What Washington means by policy reform". In J. WILLIAMSON (org.) Latin American Adjustment: How Much Has Happened? Washington: Institute for International Economics.

WOOLDRIDGE, J. M. (2001) “Applications of generalized method of moments estimation". Journal of Economic Perspectives, Vol. 15, n ${ }^{\circ}$ 4, p. 87-100.

WORLD BANK (2013) World Development Indicators. Base de Dados Online. Disponível em: http:// data.worldbank.org/data-catalog/world-development-indicators/wdi-2013 